\title{
A Nonlinear Analytical Model for the Rapid Prediction of the Torque of Synchronous Reluctance Machines
}

\author{
Giacomo Bacco, Student Member, IEEE, Nicola Bianchi, Fellow, IEEE, Hanafy Mahmoud
}

\begin{abstract}
The synchronous reluctance motor works under heavy saturation. This paper presents a nonlinear analytical model of the reluctance machine, which is used to derive both average and torque harmonics as a function of the rotor geometry. Maps showing the torque harmonics as a function of the rotor barrier angles are derived. These maps are useful tools for the machine designer to get a proper rotor geometry. The torque maps are compared with those obtained from both linear analytical and finite element models. The maps computed analytically show good agreement with those derived by means of finite element analysis, and they are obtained in a much smaller computing time.
\end{abstract}

Index Terms-Electric machines, AC motors, Design tools, Synchronous reluctance machine, Analytical model, Saturation

\section{INTRODUCTION}

B OTH synchronous reluctance (REL) and permanent magnet assisted reluctance (PMAREL) machines are more and more used, ranging from low- to medium-power applications as alternatives to the more expensive rare-earth magnet motors and to the less efficient induction motors [1]-[3]. Although the intrinsic advantages of these machines, a thoughtful design is key to reach the goals of good performance and high efficiency [4]-[6]. In particular, one of the most important design step is the choice of the number of flux-barriers and their end-angles [7], [8].

At first, the model with linear $B-H$ characteristic is described. Then the model is extended to include the saturation of some parts of the machine. Conversely to previous models, the saturation is taken into account following all the main flux lines paths inside the machine. Then a proper saturation coefficient is assigned to each of this path and applied at the air-gap. The analytical model proves to be fast and fairly accurate in any calculation. Therefore it is quick and easy to obtain the behavior of the average torque and torque ripple as a function of the rotor flux-barrier geometry. The result is presented using maps, which are essential for finding a proper combination of barrier angles which gives maximum average torque and minimum torque ripple.

Manuscript received October 12, 2017; revised January 10, 2018; accepted February 4, 2018. Paper no. TEC-00790-2017. (Corresponding author: Giacomo Bacco.) Giacomo Bacco and Nicola Bianchi are with the Electrical Drives Laboratory, Department of Industrial Engineering, University of Padova, Padova, 35131, Italy (e-mail: giacomo.bacco@phd.unipd.it nicola.bianchi@unipd.it).Hanafy Mahmoud is with the Department of Electrical and Electronic Engineering, University of Nottingham, NG72RD, Nottingham (UK), and also with the Department of Electrical Power and Machines, Cairo University, 12613 Giza, Egypt (e-mail: eng.hanafy4@yahoo.com).

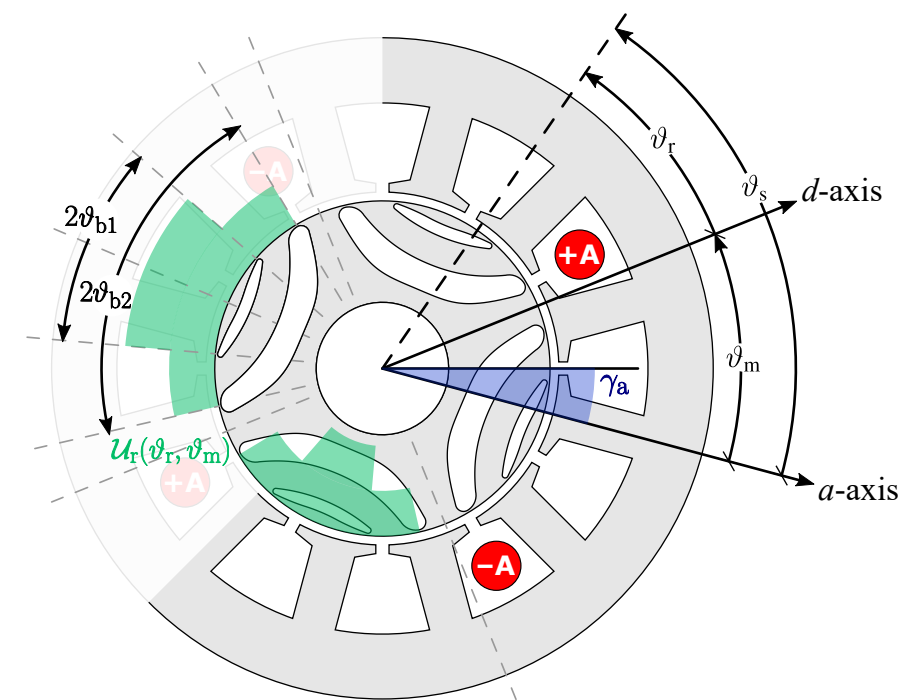

Fig. 1: Sketch of a REL motor with two flux-barriers per pole together with the reference axes and the barrier angle quotes. The colored area on top of the flux-barriers represents the rotor magnetic scalar potential distribution for half the machine.

\section{ANALYTICAL MODEL}

The analytical model considers REL machines with transversally laminated rotor. Furthermore, only integral-slot winding are considered. The electric loading of a symmetric threephase distributed winding is [8]:

$$
\mathcal{K}_{\mathrm{s}}\left(\vartheta_{\mathrm{s}}, \vartheta_{\mathrm{m}}\right)=\sum_{\substack{\nu=6 k+1 \\ k \in \mathbb{Z}}}^{\infty} \hat{K}_{\nu} \sin \left(\nu p \vartheta_{\mathrm{s}}-p \vartheta_{\mathrm{m}}-\alpha_{i}^{\mathrm{e}}\right)
$$

where $\nu$ is the space harmonic order whose values belong to the set $\{1,-5,7,-11,13, \ldots\}, \hat{K}_{\nu}$ is the amplitude of the electric loading $\nu$-th harmonic, $p$ is the number of pole pairs, $\vartheta_{\mathrm{s}}$ is the angular coordinate fixed to the stator, $\vartheta_{\mathrm{m}}$ is the rotor angular position, and $\alpha_{i}^{\mathrm{e}}$ is the current electric angle. It is worth noticing that (1) reproduces every space harmonic generated by the discretized winding.

The electric loading gives rise to the stator magnetic scalar potential, given by

$$
\mathcal{U}_{\mathrm{s}}\left(\vartheta_{\mathrm{s}}, \vartheta_{\mathrm{m}}\right)=\int \mathcal{K}_{\mathrm{s}}\left(\vartheta_{\mathrm{s}}, \vartheta_{\mathrm{m}}\right) \frac{D}{2} d \vartheta_{\mathrm{s}}
$$


where $D$ is the stator diameter at the air-gap. For a three-phase machine it is

$$
\mathcal{U}_{\mathrm{s}}\left(\vartheta_{\mathrm{s}}, \vartheta_{\mathrm{m}}\right)=-\frac{D}{2} \sum_{\substack{\nu=6 k+1 \\ k \in \mathbb{Z}}} \frac{\hat{K}_{\nu}}{p \nu} \cos \left(\nu p \vartheta_{\mathrm{s}}-p \vartheta_{\mathrm{m}}-\alpha_{i}^{\mathrm{e}}\right)
$$

Similarly, the rotor magnetic scalar potential, which reacts to the stator potential, can also be expressed by means of its Fourier series expansion

$$
\mathcal{U}_{\mathrm{r}}\left(\vartheta_{\mathrm{r}}, t\right)=\sum_{\xi=1}^{+\infty} \hat{U}_{\xi} \sin \left(\xi p \vartheta_{\mathrm{r}}\right)
$$

It is different from zero when the flux flows in the $q$-axis direction, crossing the flux-barriers. The rotor magnetic scalar potentials and the rotor barrier angles are grouped in vectors:

$$
\begin{aligned}
\boldsymbol{u}_{\mathrm{r}} & =\left\{U_{\mathrm{r} 1}, U_{\mathrm{r} 2}, \ldots, U_{\mathrm{r} n}\right\}^{\mathrm{T}}, \\
\boldsymbol{\vartheta}_{\mathrm{b}} & =\left\{\vartheta_{\mathrm{b} 1}, \vartheta_{\mathrm{b} 2}, \ldots, \vartheta_{\mathrm{b} n}\right\}^{\mathrm{T}}
\end{aligned}
$$

A quasi-diagonal matrix $\mathbf{G}$ can be built, given by

$$
\mathbf{G}=\left[\begin{array}{ccccc}
+1 & -1 & & & \\
& +1 & -1 & & \\
& & \ddots & \ddots & \\
& & & +1 & -1 \\
& & & & +1
\end{array}\right]
$$

with +1 in the main diagonal and -1 in the second right-hand diagonal, such that

$$
\hat{U}_{\xi}=\frac{4}{\pi \xi} \sin \left(\xi \frac{\pi}{2}\right)\left[\sin \left(\xi p \vartheta_{\mathrm{b}}^{\mathrm{T}}\right) \mathbf{G} \boldsymbol{u}_{\mathrm{r}}\right]
$$

where $\sin \left(\xi \boldsymbol{\vartheta}_{\mathrm{b}}^{\mathrm{T}}\right)$ is a row vector of sines, $\mathbf{G} \boldsymbol{u}_{\mathrm{r}}$ is the product of the matrix (7) and the vector $\boldsymbol{u}_{\mathrm{r}}$, defined in (5). The magnetic potentials of the islands can be calculated as the solution of the magnetic circuit shown in Fig. 2. The stator magnetic scalar potential, given in (3), can be expressed in the rotor reference frame. Since

$$
\vartheta_{\mathrm{s}}=\vartheta_{\mathrm{r}}+\vartheta_{\mathrm{m}}
$$

it results in

$$
\mathcal{U}_{\mathrm{s}}\left(\vartheta_{\mathrm{r}}, \vartheta_{\mathrm{m}}\right)=-\sum_{\substack{\nu=6 k+1 \\ k \in \mathbb{Z}}} \frac{\hat{K}_{\nu} D}{2 p \nu} \cos \left[\nu p \vartheta_{\mathrm{r}}+(\nu-1) p \vartheta_{\mathrm{m}}-\alpha_{i}^{\mathrm{e}}\right]
$$

It is split using sine and cosine as:

$$
\begin{gathered}
\mathcal{U}_{\mathrm{s}}\left(\vartheta_{\mathrm{r}}, \vartheta_{\mathrm{m}}\right)=-\sum_{\substack{\nu=6 k+1 \\
k \in \mathbb{Z}}} \frac{\hat{K}_{\nu} D}{2 p \nu}\left[\cos \left(\nu p \vartheta_{\mathrm{r}}\right) \cos \left((\nu-1) p \vartheta_{\mathrm{m}}-\alpha_{i}^{\mathrm{e}}\right)+\right. \\
\left.-\sin \left(\nu p \vartheta_{\mathrm{r}}\right) \sin \left((\nu-1) p \vartheta_{\mathrm{m}}-\alpha_{i}^{\mathrm{e}}\right)\right]
\end{gathered}
$$

$\mathcal{U}_{\mathrm{r}}\left(\vartheta_{\mathrm{r}}, \vartheta_{\mathrm{m}}\right)$ also contains harmonics multiple of three and they can be grouped apart. Then, it is easy to verify that $\mathcal{U}_{\mathrm{r}}$ is an even function with respect to the harmonic order $\xi$, so positive or negative indexes can be used indifferently.

$$
\mathcal{U}_{\mathrm{r}}\left(\vartheta_{\mathrm{r}}, \vartheta_{\mathrm{m}}\right)=\sum_{\substack{\nu=6 k+1 \\ k \in \mathbb{Z}}} \hat{U}_{\nu} \sin \left(\nu p \vartheta_{\mathrm{r}}\right)+\sum_{\substack{\mu=6 h+3 \\ h \in \mathbb{Z}^{+}}} \hat{U}_{\mu} \sin \left(\mu p \vartheta_{\mathrm{r}}\right)
$$

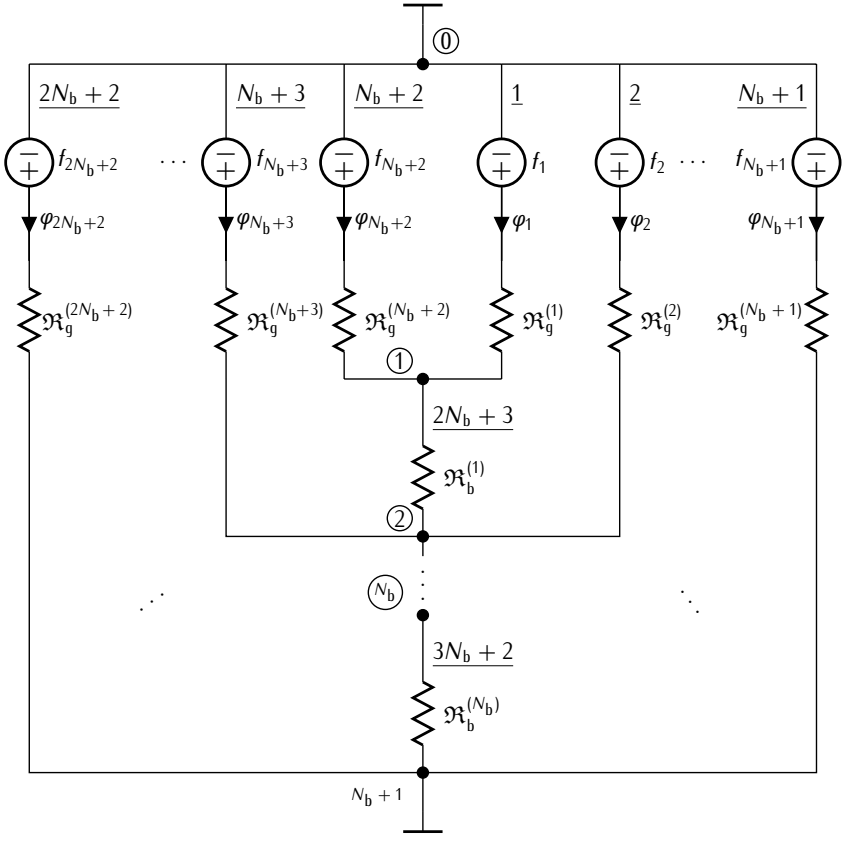

Fig. 2: Magnetic circuit of one pole of the rotor of a REL machine. Circled labels represent nodes while underlined labels represent edges of the circuit.

The air-gap flux density can be expressed as the difference of the two magnetic scalar potentials [8], [9]:

$$
\mathcal{B}_{\mathrm{g}}\left(\vartheta_{\mathrm{r}}, \vartheta_{\mathrm{m}}\right)=\mu_{0} \frac{-\mathcal{U}_{\mathrm{s}}\left(\vartheta_{\mathrm{r}}, \vartheta_{\mathrm{m}}\right)+\mathcal{U}_{\mathrm{r}}\left(\vartheta_{\mathrm{r}}, \vartheta_{\mathrm{m}}\right)}{g}
$$

Introducing (11) and (12) in (13), the air-gap flux density results in

$$
\begin{aligned}
& \mathcal{B}_{\mathrm{g}}=\sum_{\substack{\nu=6 k+1 \\
k \in \mathbb{Z}}} \frac{\mu_{0} D \hat{K}_{\nu}}{2 g p \nu}[\overbrace{\cos \left((\nu-1) \omega t-\alpha_{i}^{\mathrm{e}}\right) \cos \left(\nu p \vartheta_{\mathrm{r}}\right)}^{\text {stator even part }}\underbrace{-\sin \left((\nu-1) \omega t-\alpha_{i}^{\mathrm{e}}\right) \sin \left(\nu p \vartheta_{\mathrm{r}}\right)}_{\text {stator odd part }}]+ \\
&+\underbrace{\sum_{\substack{\nu=6 k+1 \\
k \in \mathbb{Z}}} \frac{\mu_{0}}{g \nu} \nu \hat{U}_{\nu} \sin \left(\nu p \vartheta_{\mathrm{r}}\right)}_{\text {rotor without triplen harmonics }}+\underbrace{\sum_{\substack{\mu=6 h+3 \\
h \in \mathbb{Z}^{+}}} \frac{\mu_{0}}{g \mu} \mu \hat{U}_{\mu} \sin \left(\mu p \vartheta_{\mathrm{r}}\right)}_{\text {rotor triplen harmonics }}
\end{aligned}
$$

and reordering

$$
\begin{aligned}
\mathcal{B}_{\mathrm{g}}\left(\vartheta_{\mathrm{r}}, \vartheta_{\mathrm{m}}\right) & =\sum_{\nu}\left[\frac{\alpha_{\nu}}{\nu} \cos \left(\nu p \vartheta_{\mathrm{r}}\right)+\frac{\beta_{\nu}}{\nu} \sin \left(\nu p \vartheta_{\mathrm{r}}\right)\right]+ \\
& +\sum_{\mu} \frac{\gamma_{\mu}}{\mu} \sin \left(\mu p \vartheta_{\mathrm{r}}\right)
\end{aligned}
$$

where $\alpha_{\nu}, \beta_{\nu}$ derives from the coefficients which multiply the cosine and sine functions under the summations in $\nu$, while $\gamma_{\mu}=\mu_{0} \mu \hat{U}_{\mu} / g$.

An example of the magnetic potentials and the flux density is reported in Fig. 3. 


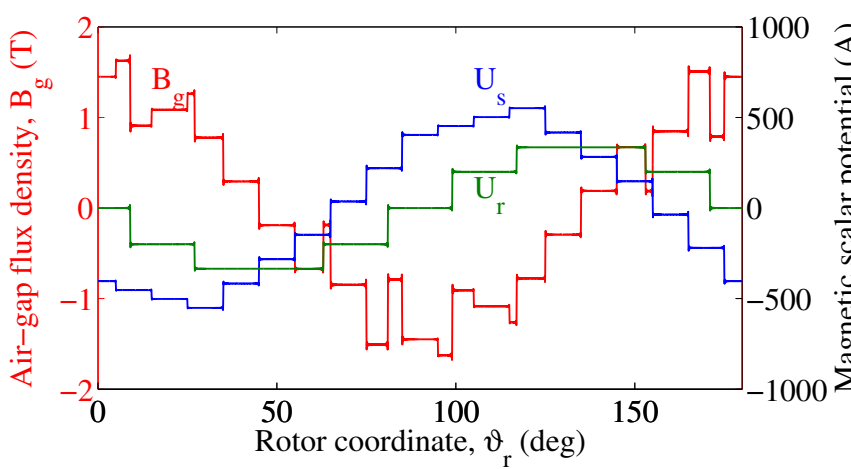

Fig. 3: Air-gap flux density and magnetic scalar potentials of a 2-barrier REL machine without saturation at $t=0$.

\section{A. Torque derivation}

The torque is obtained integrating the Lorentz's force density $\mathcal{K}_{\mathrm{s}} \mathcal{B}_{\mathrm{g}}$, both function of $\vartheta_{\mathrm{r}}$ and $\vartheta_{\mathrm{m}}$ (omitted hereafter), along the air-gap surface and multiplying the result by the lever-arm $D / 2$ [8]:

$$
\tau_{\mathrm{m}}\left(\vartheta_{\mathrm{m}}\right)=-\frac{D}{2} \int_{0}^{2 \pi} \mathcal{B}_{\mathrm{g}} \mathcal{K}_{\mathrm{s}} \frac{D L_{\mathrm{stk}}}{2} d \vartheta_{\mathrm{r}}
$$

Remembering (13), it can be derived

$$
\tau_{\mathrm{m}}=\frac{\mu_{0} D^{2} L_{\mathrm{stk}}}{4 g}[\underbrace{\int_{0}^{2 \pi} \mathcal{U}_{\mathrm{s}} \mathcal{K}_{\mathrm{s}} d \vartheta_{\mathrm{r}}}_{A}-\underbrace{\int_{0}^{2 \pi} \mathcal{U}_{\mathrm{r}} \mathcal{K}_{\mathrm{s}} d \vartheta_{\mathrm{r}}}_{B}]
$$

The first integral, labeled as $A$, is zero since $\mathcal{U}_{\mathrm{s}}$ and $\mathcal{K}_{\mathrm{s}}$ are orthogonal functions. Therefore the torque is only due to the interaction of electric loading $\mathcal{K}_{\mathrm{s}}$ and the magnetic scalar potential of the rotor $\mathcal{U}_{\mathrm{r}}$.

The final expression of the torque is

$$
\begin{aligned}
\tau_{\mathrm{m}}\left(\vartheta_{\mathrm{m}}\right)=- & \frac{\mu_{0} D^{2} L_{\mathrm{stk}}}{g} \sum_{\nu, k} \frac{\hat{K}_{\nu}}{\nu} \sin \nu \frac{\pi}{2} . \\
& \cdot \cos \left((\nu-1) p \vartheta_{\mathrm{m}}-\alpha_{i}^{\mathrm{e}}\right)\left[\sin \left(\nu \boldsymbol{\vartheta}^{\mathrm{T}}\right) \mathbf{G} \boldsymbol{u}_{\mathrm{r}}\right]
\end{aligned}
$$

\section{B. Tooth flux density}

To compute the stator tooth flux density, the air-gap flux density $\mathcal{B}_{\mathrm{g}}\left(\vartheta_{\mathrm{r}}, \vartheta_{\mathrm{m}}\right)$ in (14) has to be referred to the stator reference frame. It is

$$
\begin{aligned}
& \mathcal{B}_{\mathrm{g}}\left(\vartheta_{\mathrm{s}}, \vartheta_{\mathrm{m}}\right)=\sum_{\nu} {\left[\frac{\alpha_{\nu}}{\nu} \cos \left(\nu p \vartheta_{\mathrm{s}}-\nu p \vartheta_{\mathrm{m}}\right)+\frac{\beta_{\nu}}{\nu} \sin \left(\nu p \vartheta_{\mathrm{s}}-\nu p \vartheta_{\mathrm{m}}\right)\right] } \\
&+\sum_{\mu} \frac{\gamma_{\mu}}{\mu} \sin \left(\mu p \vartheta_{\mathrm{s}}-\mu p \vartheta_{\mathrm{m}}\right)
\end{aligned}
$$

The stator tooth flux density can be obtained through the scaled average of the air-gap flux density [10]-[12]:

$$
B_{\mathrm{t}}\left(\vartheta_{\mathrm{m}}\right)=\frac{p_{\mathrm{s}}}{\alpha_{\mathrm{s}} w_{\mathrm{t}} k_{\mathrm{pack}}} \int_{\vartheta_{d}}^{\vartheta_{d}+\alpha_{\mathrm{s}}} \mathcal{B}_{\mathrm{g}}\left(\vartheta_{\mathrm{s}}, \vartheta_{\mathrm{m}}\right) d \vartheta_{\mathrm{s}}
$$

where $p_{\mathrm{s}}$ is the slot pitch, $\alpha_{\mathrm{s}}$ the slot angle, $w_{\mathrm{t}}$ the tooth width, and $k_{\text {pack }}$ is the ratio between the total effective iron and the stack length.

It can be demonstrated that the tooth flux density assumes $q$ different behaviors, $q$ being the number of slots per pole

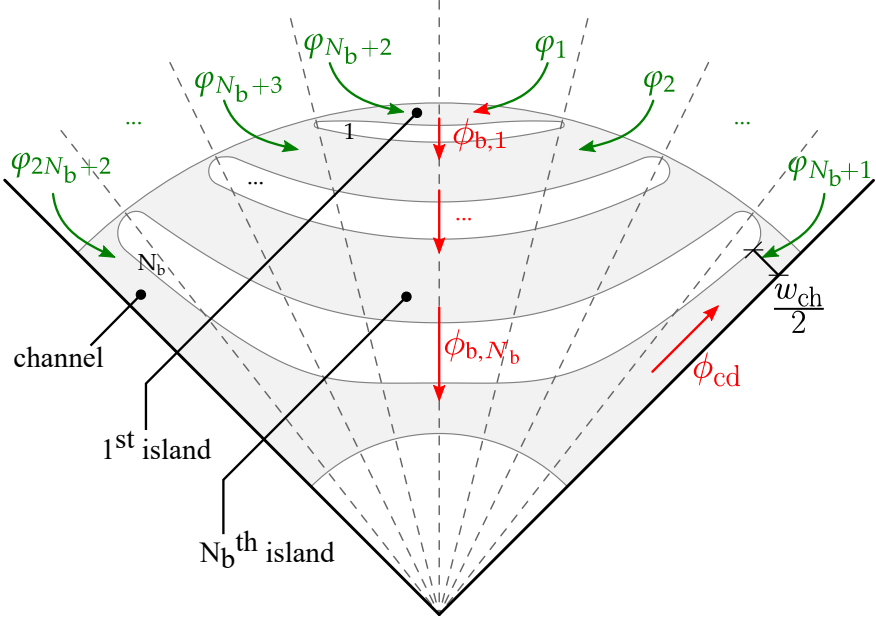

Fig. 4: Sketch of the main parts of the rotor structure and rotor fluxes direction.

and per phase, defined as $q=Q /(m 2 p)$. Thus the integration extremes can be expressed as

$$
\vartheta_{d}=(d-1) \alpha_{\mathrm{s}}-\gamma_{\mathrm{a}}, \quad d=1, \ldots, q
$$

where $\gamma_{\mathrm{a}}$ identifies the angular position of the magnetic axis of phase $a$ with respect to the first slot (see Fig. 1).

\section{Stator yoke flux density}

The flux in the stator yoke-also referred to as back-ironis obtained integrating the air-gap flux density over a pole pitch, then the flux density is

$$
\begin{aligned}
B_{\mathrm{y}}\left(\vartheta_{\mathrm{m}}\right) & =\frac{1}{2 h_{\mathrm{y}} k_{\mathrm{pack}}} \int_{-\gamma_{\mathrm{a}}}^{\frac{\pi}{p}-\gamma_{\mathrm{a}}} \mathcal{B}_{\mathrm{g}}\left(\vartheta_{\mathrm{s}}, \vartheta_{\mathrm{m}}\right) \frac{D}{2} d \vartheta_{\mathrm{s}} \\
& =\frac{w_{\mathrm{t}}}{q / 2} \sum_{\substack{\text { integer } \\
2}}^{Q / 2 p} B_{\mathrm{y}, i}\left(\vartheta_{\mathrm{m}}\right)
\end{aligned}
$$

where $h_{\mathrm{y}}=\left(D_{\mathrm{e}}-D\right) / 2$ is the stator yoke height.

\section{Rotor fluxes}

In the linear case, the fluxes entering the rotor are directly obtained from the solution of the magnetic circuit in Fig 2. Both the island and the barrier fluxes are computed. The iron path closer to the shaft is referred to as channel. The remaining iron paths for the $d$-axis flux are referred to as islands. They assume a different potential, due to the $q$-axis flux, and they are numbered accordingly to the number of the barrier beneath them. The barrier fluxes are obtained solving the magnetic network depicted in Fig. 2. The $d$-axis channel flux comes from $\phi_{\mathrm{cd}}=\varphi_{N_{\mathrm{b}}+1}-\varphi_{2 N_{\mathrm{b}}+2}$ which simply sums the two fluxes to obtain the whole direct flux of the channel. Then

$$
B_{\mathrm{cd}}=\frac{\phi_{\mathrm{cd}}}{w_{\mathrm{ch}} k_{\mathrm{pack}} L_{\mathrm{stk}}}
$$

where $w_{\mathrm{ch}} \approx\left(1-k_{\text {air }}\right) D \sin \left(\frac{\pi}{2 p}-\vartheta_{\mathrm{b} N_{\mathrm{b}}}\right)$ is an approximation of the minimum width of the channel. 
For the $q$-axis flux of the main channel, just half of the last barrier flux can be considered, with $\phi_{\mathrm{cq}}=\phi_{\mathrm{b}, N_{\mathrm{b}}} / 2$. Thus

$$
B_{\mathrm{cq}}=\frac{\phi_{\mathrm{cq}}}{l_{\mathrm{ch}} k_{\mathrm{pack}} L_{\mathrm{stk}}}
$$

where $l_{\mathrm{ch}}=\left(D_{\text {re }}-D_{\text {ri }}\right) / 2$. Then, it is

$$
\left|\boldsymbol{B}_{\mathrm{c}}\right|=\sqrt{B_{\mathrm{cd}}^{2}+B_{\mathrm{cq}}^{2}}
$$

The d-axis fluxes of the islands result directly from the magnetic circuit fluxes, as

$$
B_{\mathrm{id}, j}=\frac{\varphi_{j}}{k_{\mathrm{pack}} L_{\mathrm{stk}} w_{\mathrm{isl}, j}}
$$

Regarding the $q$-axis fluxes of the islands, the average of the fluxes above and beneath each island is computed. An average cross-section area of each island is considered, so that

$$
\begin{aligned}
& \vartheta_{\mathrm{isl}, j}=\frac{\vartheta_{\mathrm{b}, j}+\vartheta_{\mathrm{b}, j-1}}{2} \\
& l_{\text {isl }, j}=\vartheta_{\text {isl }, j} D \\
& \left(\begin{array}{c}
\phi_{\mathrm{iq}, 1} \\
\phi_{\mathrm{iq}, 2} \\
\phi_{\mathrm{iq}, 3} \\
\vdots \\
\phi_{\mathrm{iq}, N_{b}}
\end{array}\right)=\frac{1}{2}\left[\begin{array}{ccccc}
2 & & & \\
1 & 1 & & & \\
& 1 & 1 & & \\
& & \ddots & \ddots & \\
& & & 1 & 1
\end{array}\right]\left(\begin{array}{c}
\phi_{\mathrm{b} 1} \\
\phi_{\mathrm{b} 2} \\
\phi_{\mathrm{b} 3} \\
\vdots \\
\phi_{\mathrm{b} N_{b}}
\end{array}\right) \\
& B_{\mathrm{iq}, j}=\frac{\phi_{\mathrm{iq}, j}}{k_{\mathrm{pack}} L_{\mathrm{stk}} l_{\mathrm{isl}, j}} \\
& \left|\boldsymbol{B}_{\mathrm{i}, j}\right|=\sqrt{B_{\mathrm{id}, j}^{2}+B_{\mathrm{iq}, j}^{2}}
\end{aligned}
$$

\section{ANALYTICAL MODEL WITH SATURATION}

The previous analytical model works properly in case of linear behaviour of ferromagnetic material, that is, in the first part of the iron $B-H$ curve. However, this condition is barely met. In REL motors the rotor iron paths are saturated to achieve a quite high torque density and power factor. Thus, in practice, the saturation of the machine has to be taken into account.

Hereafter, the saturation is taken into account through a discretization of the flux line paths into sections. In particular, a section can be a stator tooth, a stator yoke sector, or a rotor half iron path. The path of a flux line results a succession of these sections. Therefore a magnetic voltage drop and a saturation factor are associated with each flux line and referred to the air-gap. At first the rotor iron paths are considered, and then the stator teeth and yoke.

\section{A. Rotor channel and islands magnetic voltage drop}

The magnetic voltage drop of the rotor channel is estimated from (24). Since the flux density is computed in the smallest cross-section, it is the maximum value of the channel. The average is estimated as $B_{\mathrm{c}}^{\star}=0.85\left|\boldsymbol{B}_{\mathrm{c}}\right|$ and, from the iron $B$ $H$ curve, the magnetic field $H_{\mathrm{c}}$ is obtained (in the following, it is indicated as $B \rightarrow H$ ). Then the magnetic voltage drop results $\psi_{\mathrm{c}}=H_{\mathrm{c}} l_{\mathrm{ch}}$. Similarly, the islands have a complex geometry and a different flux density in every point. The middle section of the island is considered, where the width is precisely known. It should be noted that the two half-island paths have different voltage drops due to part of the entering elementary flux that crosses the barrier and does not reclose on the other side. To recover this difference, a magnetic voltage drop is computed for every half island using the elementary flux.The procedure is sketched as follows:

$$
\left|\boldsymbol{B}_{\mathrm{i}, j}\right| \longrightarrow H_{\mathrm{i}, j} \quad \text { then } \quad \psi_{\mathrm{i}, j}=H_{\mathrm{i}, j} \frac{l_{\mathrm{isl}, j}}{2}
$$

The same procedure is carried out for the second (South) half pole.

The vector of rotor magnetic voltage drops, corresponding to the sequence of channel and islands starting from the origin of $\vartheta_{\mathrm{r}}$, is:

$$
\boldsymbol{\psi}_{\mathrm{rp}}=\{\psi_{\mathrm{c}}, \underbrace{\psi_{\mathrm{i}, N_{\mathrm{b}}}, \ldots, \psi_{\mathrm{i}, 1}}_{\boldsymbol{\psi}_{\mathrm{i}}^{\mathrm{N}}} \underbrace{\psi_{\mathrm{i}, N_{\mathrm{b}}+2, \ldots, \psi_{\mathrm{i}, 2 N_{\mathrm{b}}+2}}}_{\boldsymbol{\psi}_{\mathrm{i}}^{\mathrm{S}}}, \psi_{\mathrm{c}}\}^{\mathrm{T}}
$$

where $\psi_{\mathrm{i}}^{\mathrm{N}}$ and $\boldsymbol{\psi}_{\mathrm{i}}^{\mathrm{S}}$ are the vectors of the first half pole (identified by the superscript $\mathrm{N}$ meaning North) and the second half pole (identified by the superscript $\mathrm{S}$ meaning South) voltage drops, respectively.

Numerically, this vector is distributed into a number of points accordingly to the angle spanned. In the end, the distribution of the rotor magnetic voltage drops is equivalent to the distribution of the scalar magnetic potentials along the air-gap.

$$
\psi_{\mathrm{r}}=\{\underbrace{\underbrace{\psi_{\mathrm{c}}, \ldots, \psi_{\mathrm{c}}}_{N_{N_{\mathrm{b}}}+1}, \underbrace{\psi_{\mathrm{i}, N_{\mathrm{b}}}, \ldots, \psi_{\mathrm{i}, N_{\mathrm{b}}}}_{N_{N_{\mathrm{b}}}}, \ldots, \underbrace{\psi_{\mathrm{i}, 1}, \ldots, \psi_{\mathrm{i}, 1}}_{N_{1}}, \ldots\}^{\mathrm{T}}}_{\text {Res } / 2 p}
$$

where Res is the number of points used for discretizing the space $[0,2 \pi]$ along the air-gap, and the subscript of $N$ refers to the corresponding island.

\section{B. Stator teeth and yoke magnetic voltage drops}

The instantaneous tooth flux density in the saturation case is obtained through the numerical integration of (19). Once the flux density for every tooth of interest (typically $Q / p$ teeth for an integer-slot winding) is computed, the magnetic voltage drops are derived, as follows:

$$
\left|B_{\mathrm{t}, j}\right| \longrightarrow H_{\mathrm{t}, j} \quad \text { then } \quad \psi_{\mathrm{t}, j}=H_{\mathrm{t}, j} h_{\mathrm{s}}
$$

where $h_{\mathrm{s}}$ is the tooth height.

The back-iron corresponding to a pole pair is split into $Q / p$ parts, each one covering a slot angle, as illustrated in Fig. 5 (dotted lines). The flux density can be derived for each part, as

$$
\begin{aligned}
& \tilde{\phi}_{\mathrm{y}}(k)=w_{\mathrm{t}} L_{\mathrm{stk}} \sum_{j=1}^{k} B_{\mathrm{t}, j} \quad k=1, \ldots, Q / p \\
& \phi_{\mathrm{y}}(k)=\tilde{\phi}_{\mathrm{y}}(k)-\frac{1}{Q / p} \sum_{j=1}^{Q / p} \tilde{\phi}_{\mathrm{y}}(j) \\
& B_{\mathrm{y}}(k)=\frac{\left|\phi_{\mathrm{y}}(k)\right|}{h_{\mathrm{y}} L_{\mathrm{stk}}} \longrightarrow H_{\mathrm{y}}(k), \quad \psi_{\mathrm{y}}(k)=H_{\mathrm{y}}(k) \Delta l
\end{aligned}
$$




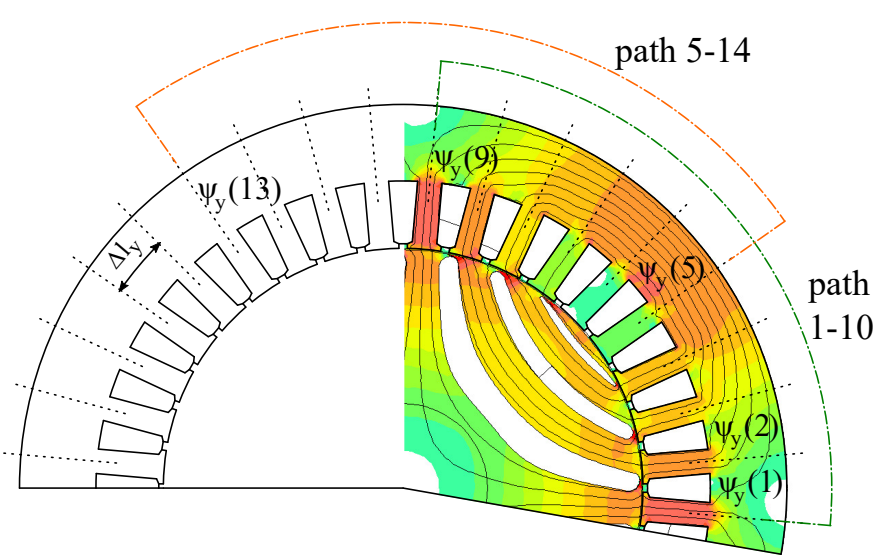

Fig. 5: Real flux lines and main integration paths $(Q=$ $36,2 p=4)$.

where $\Delta l=\pi\left(D_{\mathrm{e}}-h_{\mathrm{y}}\right) / Q$ is the average length of one backiron part. Once the stator yoke voltage drops are computed in any section, the flux path for all flux lines of interest have to be correctly identified. Two of these lines are shown in Fig. 5. Summing the voltage drops along these lines is equivalent to consider that each flux line flows for a full pole and it encounters $Q / 2 p$ voltage drops along its path. The voltage drops are summed with the sign of the corresponding flux in the same part. Finally, every flux line has gathered the same overall magnetic voltage drop of the actual flux line of that particular tooth. Therefore, it is

$$
\psi_{\mathrm{yt}}(n)=\left|\frac{1}{2} \sum_{k=n}^{n+\frac{Q}{2 p}-1} \psi_{\mathrm{y}}(k) \operatorname{sign} \phi_{\mathrm{y}}(k)\right|, n=1, \ldots, Q / 2 p
$$

where the double subscript ' $y t$ ' indicates that the yoke drop is referred to the tooth. The $\frac{1}{2}$ occurs since half the path is counted. For example, let us consider the path 1-10 shown in Fig. 5, which covers $\psi_{\mathrm{y}}(1)$ to $\psi_{\mathrm{y}}(9)$ : it starts from tooth number 1 (below the first slot) and it ends in tooth 10 . The total magnetic voltage drop associated to this line is

$$
2 \psi_{\mathrm{yt}}(1)=\psi_{\mathrm{y}}(1)+\psi_{\mathrm{y}}(2)+\ldots+\psi_{\mathrm{y}}(9)
$$

On the other hand, along the path $5-14$ it is

$$
\begin{aligned}
2 \psi_{\mathrm{yt}}(5) & =\psi_{\mathrm{y}}(5)+\ldots+\psi_{\mathrm{y}}(9)-\psi_{\mathrm{y}}(10)-\ldots-\psi_{\mathrm{y}}(13) \\
& \cong \psi_{\mathrm{y}}(5)
\end{aligned}
$$

The last equivalence is the main assumption of this computation. In fact it implies that $\psi_{\mathrm{y}}(6)$ is equal to $\psi_{\mathrm{y}}(13)$ and so on, and the remaining term is just $\psi_{\mathrm{y}}(5)$. Even if the machine is not symmetrically magnetized, this is a fairly good approximation thanks to the lower value of the $q$-axis flux with respect to the $d$-axis flux. Even with high values of $\alpha_{i}^{\mathrm{e}}$ it can be shown that this approximation is good enough.

Similarly to the rotor, the stator magnetic voltage drops can be collected and distributed along the discretization of the angular coordinate at the air-gap.

\section{Total magnetic voltage drop and saturation factor}

In order to combine the rotor and stator magnetic voltage drop distributions, the rotor position and the first tooth displacement have to be taken into account through the shift of one of the two distributions, according to the adopted reference frame. The air-gap magnetic voltage drop can be easily computed from the flux density obtained in the previous iteration.

$$
\boldsymbol{\Psi}_{\mathrm{g}}=\boldsymbol{H}_{\mathrm{g}} g=\frac{\boldsymbol{B}_{\mathrm{g}}}{\mu_{0}} g
$$

All the magnetic voltage drops are summed to obtain the total voltage drop, which is again referred to the air-gap.

$$
\Psi_{\text {tot }}=\Psi_{\mathrm{g}}+\Psi_{\mathrm{s}}^{\mathrm{r}}+\boldsymbol{\Psi}_{\mathrm{r}}
$$

where $\Psi_{\mathrm{s}}^{\mathrm{r}}$ is the vector of stator voltage drops in the rotor reference frame. Then

$$
\boldsymbol{k}_{\mathrm{sat}}=\frac{\boldsymbol{\Psi}_{\mathrm{tot}}}{\boldsymbol{\Psi}_{\mathrm{g}}}
$$

This saturation factor is different from the usually adopted factor. In fact (41) is a distribution of saturation factors along the air-gap that better represents the saturation of the machine.

The adopted iteration scheme is fixed-point like, with a random relaxation to improve the stability of the convergence:

$$
\boldsymbol{k}_{\text {sat }}^{(m+1)} \leftarrow \boldsymbol{k}_{\text {sat }}^{(m)}+0.5 \text { rand }\left(\boldsymbol{k}_{\text {sat }}^{(m+1)}-\boldsymbol{k}_{\text {sat }}^{(m)}\right)
$$

For the next iteration the updated air-gap flux density is simply

$$
\boldsymbol{B}_{\mathrm{g}}^{(m+1)}=\mu_{0} \frac{-\boldsymbol{U}_{\mathrm{s}}+\boldsymbol{U}_{\mathrm{r}}}{\boldsymbol{k}_{\mathrm{sat}}^{(m+1)} g}
$$

and the iteration cycle restarts. The error of the method was evaluated through

$$
\epsilon_{\mathrm{sat}}=\left\|\boldsymbol{k}_{\mathrm{sat}}^{(m+1)}-\boldsymbol{k}_{\mathrm{sat}}^{(m)}\right\|
$$

\section{TORQUE MAPS}

The nonlinear model is used to compute the impact of the rotor geometry on both the average torque and torque ripple. In particular, the impact of the flux-barrier-end angles is analyzed, since they heavily affect the torque ripple [8]. The average torque and some torque ripple harmonics are computed as a function of the flux-barrier angles, $\vartheta_{\mathrm{b} 1}, \vartheta_{\mathrm{b} 2}$, and their amplitude is shown graphically by using maps. Fig. 6 reports such torque maps, computed for a reference motor with two flux barriers whose data is reported in Table I:

TABLE I: Parameters of the reference motor.

\begin{tabular}{ll}
\hline$Q=36$ & number of slots \\
$2 p=4$ & number of poles \\
$y_{\mathrm{q}}=9$ & coil pitch \\
$D_{\mathrm{e}}=200 \mathrm{~mm}$ & stator outer diameter \\
$D=125 \mathrm{~mm}$ & stator inner diameter \\
$L_{\text {stk }}=40 \mathrm{~mm}$ & lamination stack length \\
$g=0.35 \mathrm{~mm}$ & air-gap thickness \\
$S_{\text {slot }}=100 \mathrm{~mm}^{2}$ & slot section area \\
$J=3 \mathrm{~A} / \mathrm{mm}^{2}$ & conductor current density \\
$k_{\text {fill }}=0.45$ & slot fill factor \\
$k_{\text {pack }}=0.95$ & lamination pack factor \\
$k_{\text {air }}=0.35$ & ratio of rotor magnetic insulation \\
\hline
\end{tabular}


For the sake of an easy comparison, Fig. 6 reports three columns, which refer to the results obtained by means of:

i. the analytical linear model [8],

ii. the nonlinear model, described above,

iii. the FE method applied on the same motor geometries [13]-[15].

The results on synchronous REL motor achieved through FE analysis have been compared with experimental test several times by the authors, obtaining satisfactory agreements [8], [16]-[18] but also in other works [19]-[21]. For this reason, in the following comparison, the results computed analytically are considered to be valid if they agree with the FE results.

The first row reports the comparison among average torque maps. The map computed with the linear model shows higher average torques with respect to the other two maps. This is obvious since the model does not take into account the iron saturation. However, it is worth noticing that the behavior of the torque curves is almost identical.

Comparing the maps of the second and third column, it is possible to note that the nonlinear analytical model predicts correctly not only the behavior of the torque maps as a function of $\vartheta_{\mathrm{b} 1}$ and $\vartheta_{\mathrm{b} 2}$, but also its amplitude. Therefore, the nonlinear model can be used as an alternative to FE simulation to derive such a map.

For this machine configuration, the average torque is almost independent of the first barrier angle. It can be noted that the average torque reaches its maximum in a wide region. For instance, for $\vartheta_{\mathrm{b} 2}^{\mathrm{e}}>60^{\circ}$ the maps are quite flat. Thus the designer is free to move within this space looking for torque ripple minima. This behaviour is reflected also by the FE map.

The second row shows the maps of the torque harmonic of order 6, which is the lowest order one. Independently from the model used, it appears that there is an evident minimum-highlighted by the black dot-corresponding to the angle combination $\left(\vartheta_{\mathrm{b} 1}^{\mathrm{e}}, \vartheta_{\mathrm{b} 2}^{\mathrm{e}}\right)=\left(36^{\circ}, 72^{\circ}\right)$. Such a point is coincident in the maps obtained from the linear and nonlinear analytical models, while it is a bit shifted when FE is used. This is caused by the local saturation of tooth tips and iron parts, which is not considered in the analytical models. In addition, it can be noted that the torque ripple contours obtained by the three models are in a satisfactory agreement in the whole region.

The same considerations can be made comparing the maps for the third row, which report the torque harmonic of order 18. Such a torque harmonic corresponds to the first magnetic scalar potential (also referred to as MMF) slot harmonic. They are the MMF harmonics produced by the winding discretized inside the slots (18=36 slots/2 pole pairs), which are characterized by a winding factor equal to the fundamental one. They typically cause the highest ripple. It can be noted that the number of peaks and valleys is increased with respect to the sixth torque harmonic. This trend is general: the higher the harmonic order, the higher the number of maxima and minima. Once more, peaks and valleys predicted by the linear analytical model and nonlinear analytical models are in a good agreement with those found by FE.
Finally, in the last row the maps compare the torque total harmonic distortion (THD), defined as

$$
\mathrm{THD}=\frac{\sqrt{\sum_{h \neq 0} \hat{\tau}_{h}^{2}}}{\tau_{0}}
$$

where $\tau_{0}$ is the average torque. The filled map shows THD contours, while the white superimposed contours refer to the average torque map. The brighter the color, the higher the THD. Thus, as far as the torque ripple is concerned, the better combinations of $\left(\vartheta_{\mathrm{b} 1}^{\mathrm{e}}, \vartheta_{\mathrm{b} 2}^{\mathrm{e}}\right)$ are those corresponding to the darker areas.

Furthermore, by comparing the results of the third and fourth rows, it can be observed that bright colors correspond to the peaks of torque harmonic of $18^{\text {th }}$ order, which is due to the MMF slot harmonics. This highlights the heavy impact of the MMF slot harmonics on the overall torque ripple.

Finally, as an overall conclusion, the linear and nonlinear analytical models produce a behavior of average torque map very similar to that obtained through $\mathrm{FE}$, with the difference that the linear map has higher values than the other two models. Therefore, the nonlinear analytical model has to be adopted to predict the average torque. On the other hand, both the analytical models are able to find the position of maxima and minima of the torque THD. Thus, it can be stated that even the linear model can be used in spite of the nonlinear one when searching for the flux-barrier angle combinations exhibiting a minimum torque ripple, with the advantage of a higher speed and similar accuracy. The slight shifts of maxima and minima found by the FE maps are mainly due to the local saturation of iron, which is not taken into account in the linear analytical model.

\section{CONCLUSions}

This paper has shown an accurate nonlinear analytical model for the synchronous reluctance machine. A good agreement between analytical and FE simulations has been achieved, even in highly saturated machines.

Thanks to the speed of the analytical model, it is possible to quickly obtain some maps of torque harmonics as a function of the barrier-end angles. These maps can be a useful design tool for the design of a reluctance machine. Overall, the analytical maps are able to properly estimate the FE maps.

The average torque behavior is correctly predicted by the nonlinear analytical model, while the linear model overestimates it. However, even if there are some differences in the amplitude prediction, the angle combinations corresponding to the minima and maxima are correctly estimated, by means of both the linear and nonlinear models. This fact is quite significant because it suggests that the analytical linear model can be employed to get good design points in the barrier-angle plane for a specific motor in a small amount of time (some minutes). On the other hand, the nonlinear analytical model is used to properly predict the average torque.

\section{REFERENCES}

[1] T. A. Lipo, "Synchronous Reluctance Machines-A Viable Alternative for AC Drives?" Electric Machines \& Power Systems, vol. 19, pp. 659-671, 1991. 

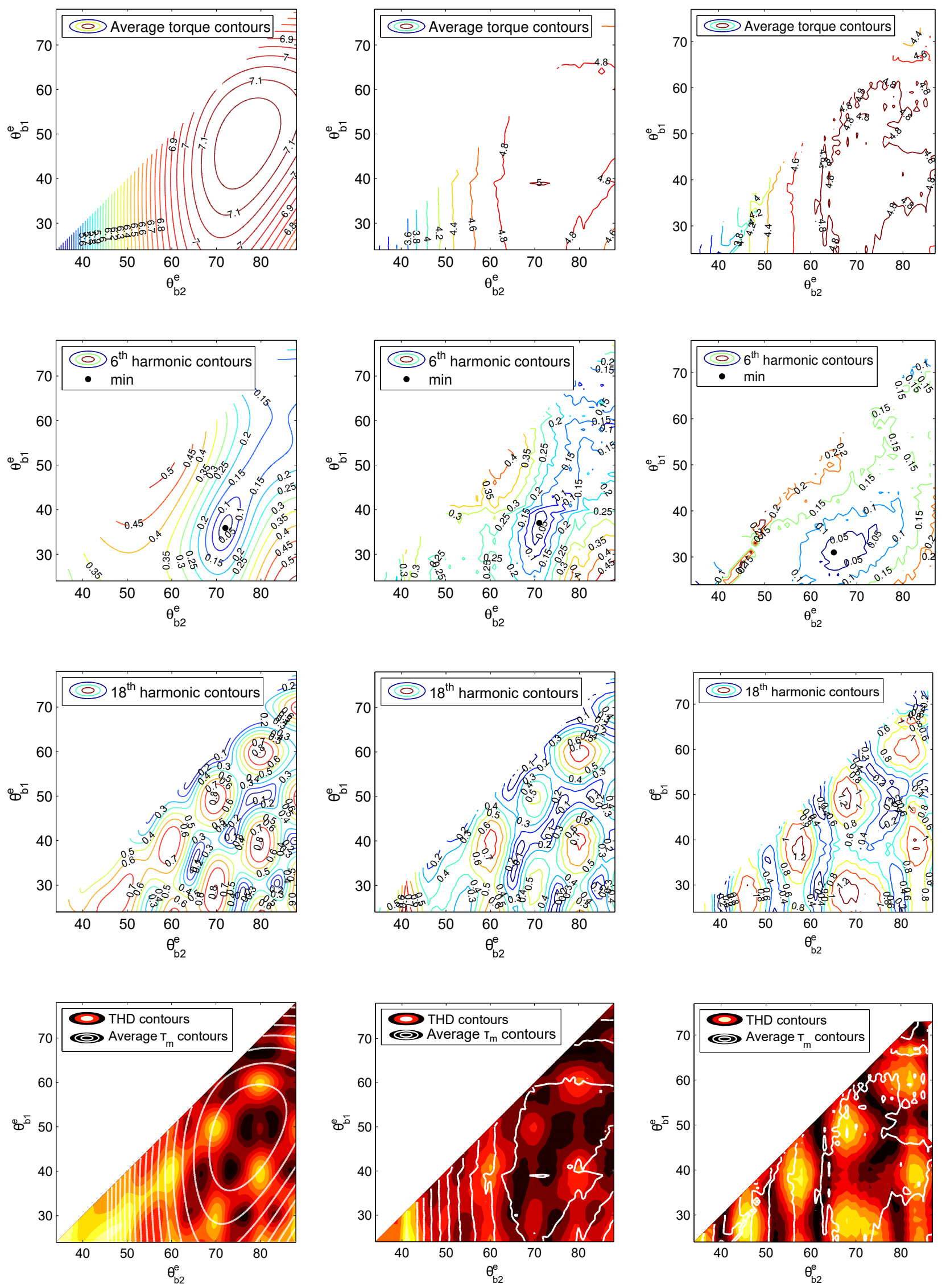

(i) Analytic linear

(ii) Analytic saturation

(iii) $\mathrm{FE}$

Fig. 6: Main torque maps of the 2-barrier rotor. The darker and cooler colors represent the valleys of the corresponding quantity. 
[2] T. A. Lipo, A. Vagati, T. Miller, L. Malesani, I. Boldea, and T. Fukao, Synchronous Reluctance Motors and Drives - A New Alternative, tutorial $\mathrm{n}$ ed., A. Vagati and T. A. Lipo, Eds. Denver: IEEE Industry Applications Society, 1994.

[3] M. J. Kamper, "Reluctance Synchronous Machine Drives - a Viable Alternative ?" in IEEE Joint IAS/PELS/IES Chapter Meeting. Graz (Austria), no. July, 2013.

[4] D. Staton, T. Miller, and S. Wood, "Maximising the saliency ratio of the synchronous reluctance motor," IET Electric Power Applications, vol. 140, no. 4, pp. 249-259, 1993.

[5] T. Matsuo and T. A. Lipo, "Rotor design optimization of synchronous reluctance machine," IEEE Transactions on Energy Conversion, vol. 9, no. 2, pp. 359-365, 1994.

[6] W. Zhao, D. Chen, T. A. Lipo, and B. I. Kwon, "Performance Improvement of Ferrite-Assisted Synchronous Reluctance Machines Using Asymmetrical Rotor Configurations," IEEE Transactions on Magnetics, vol. 51, no. 11, pp. 1-4, 2015.

[7] A. Vagati, G. Franceschini, I. Marongiu, and G. Troglia, "Design criteria of high performance synchronous reluctance motors," Conference Record of the 1992 IEEE Industry Applications Society Annual Meeting, pp. 66-73, 1992.

[8] N. Bianchi, S. Bolognani, D. Bon, and M. D. Pré, "Rotor flux-barrier design for torque ripple reduction in synchronous reluctance and PMassisted synchronous reluctance motors," IEEE Transactions on Industry Applications, vol. 45, no. 3, pp. 921-928, 2009.

[9] N. Bianchi, "Analysis of the IPM motor - Part I," in Design, Analysis, and Control of Interior PM Synchronous Machines, Tutorial Course Notes IAS, 1st ed., N. Bianchi and T. M. Jahns, Eds. Seattle: CLEUP Padova, 2006, ch. 3 .

[10] N. Bianchi and M. Barcaro, "Iron losses reduction in synchronous motors with anisotropic rotor," in Proceedings - 34th Annual Conference of the IEEE Industrial Electronics Society, IECON 2008, no. 1, Orlando, FL, 2008, pp. 1258-1263.

[11] S.-H. Han, W. Soong, and T. Jahns, "An Analytical Design Approach for Reducing Stator Iron Losses in Interior PM Synchronous Machines During Flux-Weakening Operation," in 2007 IEEE Industry Applications Annual Meeting, New Orleans, 2007, pp. 103-110.

[12] G. Pellegrino, P. Guglielmi, A. Vagati, and F. Villata, "Core losses and torque ripple in IPM machines: Dedicated modeling and design tradeoff," IEEE Transactions on Industry Applications, vol. 46, no. 6, pp. 2381-2391, 2010

[13] P. P. Silvester and R. L. Ferrari, Finite Elements for Electrical Engineers. Cambridge University Press, 1996.

[14] N. Bianchi, Electrical Machine Analysis Using Finite Elements, ser Power Electronics and Applications. Boca Raton: CRC Press, 2005.

[15] S. Salon, Finite Element Analysis of Electrical Machines, ser. Power Electronics and Power Systems. Springer US, 2012.

[16] N. Bianchi, E. Fornasiero, E. Carraro, S. Bolognani, and M. Castiello, "Electric vehicle traction based on a PM assisted synchronous reluctance motor," in 2014 IEEE International Electric Vehicle Conference, IEVC 2014, Florence, 2014, pp. 6-11.

[17] N. Bianchi, E. Fornasiero, M. Ferrari, and M. Castiello, "Experimental comparison of PM assisted synchronous reluctance motors," IEEE Transactions on Industry Applications, vol. 52, no. 1, pp. 163-171, 2016.

[18] M. Ferrari, N. Bianchi, and E. Fornasiero, "Analysis of rotor saturation in synchronous reluctance and PM-assisted reluctance motors," IEEE Transactions on Industry Applications, vol. 51, no. 1, pp. 169-177, 2015.
[19] A. Vagati, M. Pastorelli, G. Franceschini, and S. C. Petrache, "Design of low-torque-ripple synchronous reluctance motors," IEEE Transactions on Industry Applications, vol. 34, no. 4, pp. 758-765, 1998.

[20] R. R. Moghaddam, "Synchronous Reluctance Machine (SynRM) in Variable Speed Drives (VSD) Applications," $\mathrm{PhD}$ Thesis, The Royal Institute of Technology, Stockholm, 2011. [Online]. Available: http://www.diva-portal.org/smash/record.jsf?pid=diva2:417890

[21] M. Gamba, "Design of non conventional Synchronous Reluctance machine," PhD Thesis, Politecnico di Torino, 2017.

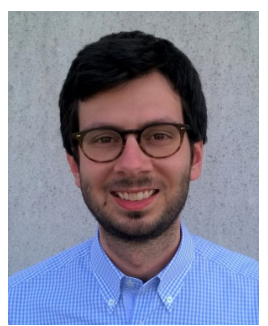

Giacomo Bacco (S'17) received both the BS in Energy Engineering in 2013 and the MS in Electrical Engineering in 2016 from the University of Padova, Padova, Italy. Currently he is a $\mathrm{PhD}$ student of the Department of Industrial Engineering at the same university. His research interests are focused in the analysis and design of synchronous machines, in particular synchronous reluctance (REL) motors.

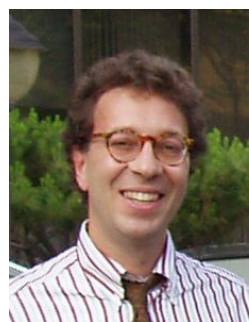

Nicola Bianchi (M'98-SM'09-FM'14) received the M.Sc. and Ph.D. degrees in electrical engineering from the University of Padova, Padova, Italy, in 1991 and 1995, respectively. In 1998, he joined the Department of Electrical Engineering, University of Padova, as an Assistant Professor, and where since 2005, he has been an Associate Professor of electrical machines, converters, and drives in the Electric Drive Laboratory, Department of Electrical Engineering. He is the author or coauthor of several scientific papers and international books on electrical machines and drives. His research interest include the field of design of electrical machines, particularly for drive applications, in which he is responsible for various projects for local and foreign industries. Prof. Bianchi has received five awards for best conference and journal papers. He is a member of the Electric Machines Committee and the Electrical Drives Committee of the IEEE Industry Applications Society. He served as a Technical Program Chair for the IEEE Energy Conversion Congress and Exposition 2014 and is an Associate Editor of the IEEE Transactions on Industry Applications.

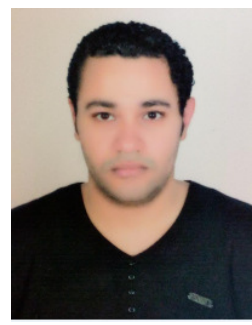

Hanafy Mahmoud received the Bachelor?s degree and the M.Sc. degree in Electrical Engineering in 2009 and 2012 respectively, from Cairo University, Cairo, Egypt. During his M.Sc. period he worked on faults detection and performance analysis of induction machines. He received the $\mathrm{PhD}$ degree from the University of Padova, Italy. Now, he is a Research Fellow at the PEMC group, Department of Electrical and Electronic Engineering, Nottingham University, UK. His research activities deal with the analysis of synchronous permanent magnet and reluctance machines, focusing on analytical and finite element modeling, faulty conditions analyses. Besides, his current research interests are high efficiency machine design, as well as analytical modeling of various electrical machines. 